\title{
Heating of the Hot Intergalactic Medium by Powerful Radio Galaxies and Associated High Energy Gamma-Ray Emission
}

\author{
Susumu Inoue $\mathrm{e}^{1,2}$ \\ inoue@th.nao.ac.jp \\ and \\ Shin Sasaki ${ }^{3}$ \\ sasaki@phys.metro-u.ac.jp
}

\begin{abstract}
There is increasing evidence that some heating mechanism in addition to gravitational shock heating may be necessary for the hot gas inside clusters and groups of galaxies in order to explain their observed X-ray scaling properties. While supernovae are the most obvious candidate heating sources, a number of recent studies have indicated that they may be energetically insufficient. Here we consider high-power, FRII radio galaxies and shock heating of the intracluster medium (ICM, including the case of the intergalactic medium prior to cluster formation) by their largescale jets. Based on the observed statistics of radio galaxies in clusters and their evolution, along with the most reasonable assumptions, it is shown that they can provide the ICM with specific energies of $1-2 \mathrm{keV}$ per particle, mainly during the redshift interval $z \sim 1-3$. This naturally meets the requirements of cluster evolution models with non-gravitational feedback in accounting for the observed deviations in the X-ray luminosity-temperature relation. In contrast to supernovae, such large-scale jets deposit their energy directly into the low density ICM outside galaxies, and are much less susceptible to radiative losses. As a clear and potentially decisive test of this scenario, we propose the observation of 'prompt' high energy gamma-rays emitted by shock-accelerated, non-thermal electrons during the epoch of ICM heating by radio galaxies, which may be feasible with the GLAST satellite. Implications for recent detections of excess hard $\mathrm{X}$-rays from groups are also discussed.
\end{abstract}

Subject headings: X-rays: galaxies: clusters, radio continuum: galaxies, galaxies: jets, intergalactic medium, cosmology, gamma-rays: theory

\section{Introduction}

Within the framework of currently popular structure formation theories, the hot, X-ray emitting intracluster medium (ICM) observed in clus-

\footnotetext{
${ }^{1}$ Division of Theoretical Astrophysics, National Astronomical Observatory, 2-21-1 Ohsawa, Mitaka, Tokyo, Japan 181-8588

${ }^{2}$ Osservatorio Astrofisico di Arcetri, Largo E. Fermi 5, I-50127, Firenze, Italy

${ }^{3}$ Department of Physics, Tokyo Metropolitan University, 1-1 Minami-Ohsawa, Hachioji, Tokyo, Japan 192-0397
}

ters and groups of galaxies (hereafter collectively referred to as 'clusters', unless otherwise specified) arises when baryonic gas falls into the gravitational potential wells of hierarchically merging dark matter halos, and is shock heated to near the corresponding virial temperature. Kaiser (1986) predicted that if the structure and evolutionary behavior of X-ray clusters are governed solely by gravitational processes, they should be 'selfsimilar' and their physical properties should obey well-defined scaling laws. Detailed numerical simulations (e.g. Eke, Navarro \& Frenk 1998) also 
agree with this prediction.

However, observational evidence has been mounting that this is not the whole story. Significant deviations from self-similarity are observed, most conspicuously in the X-ray luminositytemperature (L-T) relation. Whereas the selfsimilar model predicts the X-ray luminosity $L$ to scale with temperature $T$ as $L \propto T^{2}$, the observed relation is considerably steeper, being close to $L \propto T^{3}$ for $T \gtrsim 2 \mathrm{keV}$ clusters (e.g. David et al. 1993, Markevitch 1998, Arnaud \& Evrard 1999), and perhaps even steeper for lower $T$ groups (Ponman et al. 1996, Helsdon \& Ponman 2000). Deviations have also been discovered in the entropytemperature (S-T) relation, with evidence of an excess 'entropy floor' in objects of $T \lesssim 2 \mathrm{keV}$ (Ponman, Cannon \& Navarro 1999, Lloyd-Davies, Ponman \& Cannon 2000).

A plausible solution to this discrepancy is that the ICM (or the intergalactic medium prior to cluster formation which eventually becomes the present day ICM; also designated 'ICM' for brevity) has been shock heated by some type of energy source in addition to gravitational heating (Kaiser 1991, Evrard \& Henry 1991, for an alternative view, see Bryan 2000). This process, while having little effect for the most massive and hottest clusters, can significantly modify lower $T$ systems, raising their temperature as well as decreasing their central gas densities from the selfsimilar expectations, and thereby bringing them into agreement with the observed scaling relations. The most widely discussed energy source to date has been supernovae (SNe) (e.g. Ponman et al. 1999, Menci \& Cavaliere 2000, Cavaliere, Giacconi \& Menci 2000). As the ICM is observed to contain sub-solar abundances of $\mathrm{Fe}$ and other heavy elements, it is certain that metals produced by $\mathrm{SNe}$ inside cluster galaxies have been ejected into the ICM by some process, perhaps in the form of SN-driven 'galactic winds' (e.g. Finoguenov, David \& Ponman 2000, Renzini 2000 and references therein). However, a number of recent studies have brought into question the effectiveness of SNe as energy sources for the ICM. Employing semi-analytic models of cluster evolution, Valageas \& Silk 1999, Wu, Fabian \& Nulsen 2000 and Bower et al. 2000 have all shown that in order to reproduce the L-T relation, the non-gravitational heat input must amount to $0.5-$
$3 \mathrm{keV}$ per particle. For supernovae, this implies an extremely high efficiency of energy conversion, close to $100 \%$, which is unrealistic considering that a large fraction of the initial energy is likely to be lost radiatively (Kravtsov \& Yepes 2000). Some of the above papers have discussed the possibility that active galactic nuclei are instead the true heating agents of the ICM, but none have attempted a quantitative estimation of such an effect.

A related problem regards the soft X-ray background emission from the intergalactic medium (IGM), including regions outside virialized clusters. Recent observational estimates (Fukugita, Hogan \& Peebles 1998) as well as numerical simulations (e.g. Cen \& Ostriker 1999, Davé et al. 2000) suggest that the majority of the baryons in the universe today exists in a warm/hot IGM at temperatures $T \sim 10^{5}-10^{7} \mathrm{~K}$. Concerns have been raised that the thermal emission from such regions may exceed the observed soft X-ray background in the absence of non-gravitational heating (Pen 1999, Wu, Fabian \& Nulsen 1999, Voit \& Bryan 2001, Bryan \& Voit 2001). Again, the level of heating required to reconcile theory with observations may be of the order of a few $\mathrm{keV}$ per particle for the IGM. However, this issue is currently controversial, as the inferences seem to depend critically on the numerical resolution and treatment of cooling in the calculations (Bryan \& Voit 2001 and references therein).

We consider in this work high-power, FRII radio galaxies (RGs) and shock heating of the ICM/IGM by their large-scale jets. (The term 'radio galaxy' is used here to connote all sources with strong jets including radio-loud quasars, which are believed to be intrinsically similar objects in the context of AGN unification schemes, Urry \& Padovani 1995.) For Cyg A, the only nearby FRII RG residing inside a cluster that has been studied in sufficient depth, a strong case can be made that the RG is driving a bow shock into the ambient ICM (Carilli \& Barthel 1996 and references therein). We wish to quantify the consequences of such effects for the ICM/IGM in a cosmological context. As detailed observational information is available on the statistics of RGs in clusters (Ledlow \& Owen 1995, 1996) as well as on the evolution of RGs out to high redshifts (Dunlop \& Peacock 1990, Willott et al. 2001), we can obtain a rea- 
sonably reliable estimate of the total energy input into the ICM by RGs (Sec.2).

We discuss the notable features and advantages of ICM heating by RGs (Sec.3.1), as well as the validity of the assumptions and uncertainties that go into the above evaluation (Sec.3.2). A crucial diagnostic with which the RG scenario may be verified and distinguished from SN heating is proposed: the observation of high energy gamma-rays emitted by shock-accelerated non-thermal electrons during the period of shock activity (Sec.3.4). Other implications, including non-thermal X-ray emission from groups, are also discussed (Sec.3.5).

A rough estimate of the RG energy input along the above lines have already been presented by Rawlings (2000). Enßlin et al. $(1997,1998)$ have pointed out the importance of RGs for the energetics of the ICM, and calculations similar to those given below were also carried out by Enßlin \& Kaiser (2000); however, their focus was on the Sunyaev-Zeldovich effect, and they did not quantify the consequences in relation to the observed X-ray properties of clusters (cf. Yamada \& Fujita 2001).

\section{Energy Budget of Radio Galaxies}

\subsection{Global Energy Output in the Uni- verse}

We first evaluate the total energy output by $R G$ jets in the universe, utilizing observational information on the evolution of the RG luminosity function (LF), and the correlation between jet kinetic power and radio luminosity of RGs. Two cosmologies are considered, $\Omega=1, \Lambda=0, h_{50}=1$ (Einstein-de Sitter, referred to as 'EdS'), and $\Omega=$ $0.3, \Lambda=0.7, h_{50}=1.4$ (referred to as 'lambda') where $H_{0}=50 h_{50} \mathrm{~km} \mathrm{~s}^{-1} \mathrm{Mpc}^{-1}$. All radio luminosities are measured in units of $\mathrm{W} \mathrm{Hz}^{-1} \mathrm{sr}^{-1}$.

The redshift evolution of steep spectrum radio sources has recently been quantified in detail by Willott et al. (2001, hereafter W01). Based on a large source sample with complete redshifts and less affected by various complicating effects due to the low frequency $(151 \mathrm{MHz})$ selection, their data constitute the most accurate such information yet obtained. Defined in terms of number of sources per unit comoving volume per unit logarithmic $151 \mathrm{MHZ}$ radio luminosity $L_{151}$ at redshift $z$, their LFs $\rho\left(L_{151}, z\right)$ were modeled as com- prising two populations, a low-luminosity one and a high-luminosity one, corresponding to sources with weak and strong emission lines, respectively. The break luminosity separating the populations, $\log L_{151} \sim 26.5$, is roughly one order of magnitude larger than the luminosity of the FRI/FRII division between the two morphological classes of RGs (Fanaroff \& Riley 1974). The evolutionary behavior of each population was assumed to be different, with three different models being considered for the high-luminosity population: Model A (symmetric Gaussian in $z$ ), Model B (Gaussian rise up to a certain $z$ and constant beyond with no cutoff), Model C (Gaussian rise plus Gaussian decline in $z$ with different widths). The low luminosity population was characterized as a power-law rise up to some $z$ and then constant beyond. W01 fit such parametrizations to the data assuming two cosmologies, $\Omega=1$ or $\Omega=0$ with $\Lambda=0, h_{50}=1$, and found that all three models in either cosmology gave acceptable fits to the data including the 'no cutoff' model B. However, we will see that the total RG energy input is determined principally by RGs in the redshift range well constrained by the data, and does not depend greatly on the uncertain high- $z$ evolution. For our lambda cosmology, the LFs were obtained by converting the $\Omega=0$ LFs of W01 using their equation (14).

Willott et al. (1999, hereafter W99), have derived a relation between the jet kinetic powers $L_{j}$ of RGs and their emitted radio luminosities at $151 \mathrm{MHz} L_{151}$ of the form $L_{j}=3 \times$ $10^{45} f_{j}\left(L_{151} / 10^{28} \mathrm{~W} \mathrm{~Hz}^{-1} \mathrm{sr}^{-1}\right)^{6 / 7} \mathrm{erg} \mathrm{s}^{-1}$. This is based on physical considerations as well as on observational constraints of hot spot advance speeds and spectral ages for the general population of FRII RGs. The important parameter $f_{j}$ takes into account uncertainties in the physical conditions inside the jet lobes (e.g. departures from equipartition, proton and low energy electron content, volume filling factor, etc.), and is expected to be in the range $f_{j} \sim 1-20$ (W99). On the other hand, jet kinetic powers have also been determined observationally for individual RGs (modulo the uncertainty factor $f_{j}$ ) by Rawlings \& Saunders (1991), and the direct correlation between $L_{j}$ and $L_{151}$ is available in Rawlings (1992) for RGs in different environments; this is shown in Fig.1. The W99 relation has the correct slope but seems to underpredict the magnitude of the observed $L_{j}$ - 
$L_{151}$ relation. We choose to adopt

$L_{j}=3 \times 10^{46} f_{j}\left(\frac{L_{151}}{10^{28} \mathrm{~W} \mathrm{~Hz}^{-1} \mathrm{sr}^{-1} h_{50}^{-2}}\right)^{6 / 7} \operatorname{erg~s}^{-1} h_{50}^{-2}$,

agreeing well with the data of RGs in group-like environments (densities $n \sim 10^{-5}-10^{-3} \mathrm{~cm}^{-3}$ ), for which we are most interested in. Note that this relation is not inconsistent with the constraints discussed in W99, and is also in line with that given by Enßlin et al. (1997) using radio luminosities at $2.7 \mathrm{GHz}$. For $f_{j}$, we take a fiducial value of $f_{j}=10$, which is supported by a number of independent observational inferences (e.g. Leahy \& Gizani 1999, Hardcastle \& Worrall 2000, Blundell \& Rawlings 2000).

Regarding individual RGs, $L_{151}$ should also depend on the RG's age even for constant $L_{j}$; however this dependence is expected to be weak (e.g. W99), allowing us to neglect this complication. The density of the ambient medium obviously affects $L_{151}$ as well, but we assume that this does not induce any systematic effects, and that the above correlation is independent of $z$ (see Sec.3.2 for justification).

Utilizing the redshift dependent LFs and the above $L_{j}-L_{151}$ correlation, we integrate over radio luminosity and cosmic time to derive the total jet energy output by RGs $U_{j}$ in the universe,

$U_{j}=\int_{z_{\min }}^{z_{\max }} d z \frac{d t}{d z} \int_{L_{151, \min }}^{L_{151, \max }} d \log L_{151} L_{j}\left(L_{151}\right) \rho\left(L_{151}, z\right)$.

(c.f. Enßlin \& Kaiser 2000). The lower and upper limits for $\log L_{151}$ were taken to be 25.5 and 30 , respectively. The lower limit corresponds to the FRI/FRII dichotomy in radio morphology, below which RGs appear to be subsonic on large scales and do not drive strong shocks into the surrounding medium (e.g. Bicknell 1995, Fabian 2001 and references therein). The upper limit on $z$ was fixed to be $z_{\max }=5$, above which Compton cooling of RG lobes by the cosmic microwave background becomes very severe (Kaiser, DennettThorpe \& Alexander 1997). However, the results are not so sensitive to these values as $U_{j}$ is mainly determined by RGs near the 'break' in the LF, $\log L_{151} \sim 26-27$, and in the redshift interval $z \sim 1-3$. The results, both cumulative (integrated from $z_{\max }$ to $z$ ) and differential (per unit $z)$, are shown in units of $\mathrm{erg}^{\mathrm{Mpc}^{-3}}$ in Figs.2 and 3 .

We see that the values of $U_{j}$ integrated to $z=0$ amount to $1-3 \times 10^{57} \mathrm{erg}_{\mathrm{Mpc}^{-3}}$, and depend little on the LF model or on cosmology. As can be seen from $d U_{j} / d z$ in Figs.2b and $3 \mathrm{~b}$, the dominant contribution to $U_{j}$ comes from sources at redshifts $z \sim 1-3$, which are well constrained by the data and leave only small uncertainties in the integrals. The numbers are also quite consistent with those obtained by Enßlin \& Kaiser (2000) using different radio LFs and jet power-radio luminosity correlations.

We may average $U_{j}$ over the present-day baryon density in the universe and obtain

$\epsilon_{\mathrm{IGM}} \sim 0.15 \frac{U_{j}}{2 \times 10^{57}{\mathrm{erg} \mathrm{Mpc}^{-3}}}\left(\frac{\Omega_{b} h_{50}^{2}}{0.06}\right)^{-1} \mathrm{keV} /$ particle

taking a mean molecular weight $\mu=0.6$. If excess energies of a few $\mathrm{keV} /$ particle turn out to be necessary to halt overproduction of the soft Xray background from the warm/hot IGM, (Sec.1), RGs seem to fall short by at least an order of magnitude, even when assuming 100\% energy transfer from RG jets to the IGM. However, more detailed calculations which account for the inhomogeneous IGM and RG clustering may be required to conclusively settle this matter.

If we consider groups in the mass range $M \sim$ $2 \times 10^{13}-2 \times 10^{14} M_{\odot}($ corresponding to $T \sim 0.5-2$ $\mathrm{keV})$ in the context of currently favored structure formation models, their typical formation redshifts are $z_{f} \sim 0.3-0.5(0.04-1.4,2 \sigma$ dispersion range) for the EdS cosmology, and $z_{f} \sim 0.6-0.8(0.1-1.9$, $2 \sigma$ dispersion range) for the lambda cosmology (Lacey \& Cole 1993, Kitayama \& Suto 1996). The main epoch of RG energy input, $z \sim 1-3$, therefore mostly precedes or coincides with the formation of low $T$ systems, and is generally consistent with the picture of 'preheating', i.e. heating of the ICM prior to halo collapse (e.g. Balogh, Babul \& Patton 1999, Tozzi \& Norman 2001), as opposed to heating after collapse and virialization (e.g. Tozzi, Scharf \& Norman 2000).

\subsection{Energy Input into the Intracluster Medium}

We now translate the above results into the thermal energy input into the ICM gas, making 
use of the local LF of RGs inside clusters. It is assumed that the redshift evolution of cluster RGs is similar to that of the whole RG population as determined by W01 (see Sec.3.2). We begin by normalizing the local LF of W01 to the LF determined for RGs inside clusters by Ledlow \& Owen (1995, 1996, hereafter respectively LO95, LO96), based on their $1.4 \mathrm{GHz}$ VLA survey of Abell clusters. Conversion between luminosities at $1.4 \mathrm{GHz}$ and $151 \mathrm{MHz}$ are done assuming a spectral energy in$\operatorname{dex} \alpha=0.8$. The LO96 univariate LF $f(L)$ is presented as the fraction of elliptical galaxies (excluding S0's) of $\mathrm{R}$ magnitude $R_{c} \leq-20.5$ detected at $1.4 \mathrm{GHz}$ per each logarithmic radio luminosity bin. We determine the normalization factor $\mathcal{F}$ (units $\left.\mathrm{Mpc}^{3}\right)$ so that the W01 local LF $\rho(L, 0)$ matches the L096 LF, $\mathcal{F} \rho(L, 0) \sim f(L)$, in the 'break' luminosity range $\log L_{151} \sim 26-27$. (The quantity $\mathcal{F}^{-1}$ reflects the density of elliptical galaxies inside clusters averaged over the universe, and $\mathcal{F} U_{j}$ corresponds to the average time-integrated jet energy input per cluster elliptical.) We obtain $\log \mathcal{F} \sim 5.4$ for $h_{50}=1(\mathrm{EdS})$ and $\log \mathcal{F} \sim 4.5$ for $h_{50}=1.4$ (lambda). The shape of the W01 local LF does not agree well with that of the LO96 LF for luminosities $\log L_{151} \lesssim 25$, but this is irrelevant for our purposes as our lower luminosity limit of integration is taken to be $\log L_{151} \sim 25.5$. We assume that this fractional LF at $z=0$ is the same for all clusters and groups within the mass range of our interest (see Sec.3.2).

It is further assumed that the number of elliptical galaxies $N_{E}$ in a cluster is proportional to its gas mass $M_{g}$. More precisely, we posit that the gas which eventually evolves into the ICM of present-day clusters contains RG hosting ellipticals with a ratio of $N_{E} / M_{g}$ similar to that observed in local rich clusters. This implies a massindependent specific energy input, being the same for small groups as well as rich clusters. Taking the Coma cluster as a reference point, the number of elliptical galaxies of $R_{c} \leq-20$ within a radius $r \leq 3.4 h_{50}^{-1} \mathrm{Mpc}$ from the cluster center is $\sim 38$ (Thompson \& Gregory 1980). The LO96 LF, when integrated over radio luminosity, gives a total fraction $\sim 0.14$ implying $\sim 5$ RG hosting ellipticals in Coma; this compares favorably with the actual number of radio ellipticals observed with the appropriate properties in the Coma cluster (Venturi, Giovannini \& Feretti
1990). The gas mass within the same radius is $M_{g} \sim 4 \times 10^{14} h_{50}^{-1} M_{\odot}$ (Fusco-Femiano \& Hughes $1994)$, so we get a reference value of $N_{E} / M_{g} \sim$ $0.95 \times 10^{-13} M_{\odot}^{-1}$.

The relativistic jet of an FRII RG should drive a strong shock into the ambient medium, heating and compressing it, but not all of the jet power can be directly conveyed outside in this manner. A major portion of the total energy should also accumulate inside the 'cocoon', the region immediately enveloping the jet lobes and filled with hot, shocked jet plasma, which is separated from the ICM by a contact discontinuity (Begelman \& Cioffi 1989). Of the total energy $E_{\mathrm{RG}}$ released during a $R$ G's lifetime, the fraction imparted to the surrounding ICM as work $E_{\mathrm{ICM}}$ and that stored inside the cocoon as internal energy $E_{c}$ can be evaluated in a simple way following Enßlin \& Kaiser (2000, hereafter EK00). After cessation of the RG activity, the cocoon should expand up to a volume $V_{c}$ where its internal pressure $p_{c}$ reaches equilibrium with that of the ICM $p_{\text {ICM }}, p_{c} \sim p_{\text {ICM }}$. The work done against the ICM can be roughly estimated to be $E_{\mathrm{ICM}} \sim p_{\mathrm{ICM}} V_{c}$. If the cocoon plasma is predominantly relativistic, $p_{c} \sim \frac{1}{3} E_{c} / V_{c}$, and since $E_{\mathrm{RG}} \sim E_{\mathrm{ICM}}+E_{c}$, we get $E_{\mathrm{ICM}} / E_{\mathrm{RG}} \sim 0.25$, whereas for primarily non-relativistic cocoon plasma, $p_{c} \sim \frac{2}{3} E_{c} / V_{c}$ and $E_{\mathrm{ICM}} / E_{\mathrm{RG}} \sim 0.4$. As explained below (Sec.3.1), the ICM gas shocked by the large-scale jets of FRII RGs should generally be hot and rarefied enough for radiative cooling to be negligible, and no further reduction in efficiency is expected. We conservatively adopt $\xi_{s}=0.2$ for the fraction of the total RG energy output imparted to the ICM. (This value could be larger for reasons discussed in Sec.3.2.)

Putting all of this together, the specific energy input into the ICM $\epsilon_{\mathrm{ICM}}$ is

$$
\begin{aligned}
\epsilon_{\mathrm{ICM}} & =\xi_{s} \mathcal{F} U_{j} \mu m_{p} N_{E} / M_{g} \\
& \sim 1.2 \frac{\xi_{s}}{0.2} \frac{\mathcal{F}}{10^{5} \mathrm{Mpc}^{3}} \frac{U_{j}}{2 \times 10^{57} \mathrm{erg} \mathrm{Mpc}^{-3}} \\
& \times \frac{N_{E} / M_{g}}{0.95 \times 10^{-13} M_{\odot}^{-1}} \mathrm{keV} / \text { particle }
\end{aligned}
$$

using $\mu=0.6$. In Figs.2a and 3a, the right axes denote the values of $\epsilon_{\mathrm{ICM}}$ calculated for the different LF models. We see that with reasonable assumptions, the total energy input into the ICM by RGs 
lies in the range $\sim 1-2 \mathrm{keV}$ per particle (and could be even higher, see Sec.3.2). According to recent detailed models of cluster evolution incorporating non-gravitational feedback (Valageas \& Silk 1999, Wu, Fabian \& Nulsen 1998, 2000, Bower et al. 2000), a few keV per particle is just the amount of excess energy input required to account for the observed deviations from self-similarity in X-ray clusters.

\section{Discussion}

\subsection{On Heating of the Intracluster Medium by Radio Galaxies}

We discuss some important issues regarding shock heating of the ICM by powerful RGs. First, a single FRII RG is capable of sweeping out and heating a volume of order $(\mathrm{Mpc})^{3}$ during its lifetime. This is enough to affect a major fraction of the total ICM volume, especially for relatively low $T$ clusters and groups. We also mention that RGs are observed to possess a more centrally concentrated spatial distribution relative to normal galaxies, at least for relatively rich clusters (LO95).

Next, we emphasize the crucial advantage of RGs compared to SNe for ICM heating. The jets of high-power, FRII RGs are known to transport their energy efficiently out to regions well outside their host galaxies, and then deposit it directly into the low-density, large-scale ICM. The shocked gas is expected to possess a sufficiently low density and high temperature such that its radiative cooling time is much longer than the Hubble time (Heinz, Reynolds \& Begelman 1998, Kaiser \& Alexander 1999), possibly apart from the innermost regions of rich clusters constituting a minor fraction of the whole ICM (Tozzi \& Norman 2001). This is in stark contrast to SNe, which must first explode into the dense interstellar medium of their host galaxies where the cooling time is much shorter (see however Sasaki 2001), and thereby lose a major fraction of their energy as escaping radiation (Kravtsov \& Yepes 2000, Wu et al. 2000).

In relation to the above two points, we note that the lifetimes of individual FRII RGs are believed to be $\lesssim 10^{8}$ yr (e.g. Blundell, Rawlings \& Willott 1999), much shorter than the main duration of activity for the FRII population as a whole, as well as the typical ages of clusters. By virtue of the long cooling time of the shocked ICM, any FRII activity in a cluster's past will have left its mark on the ICM, but need not have any direct correspondence with the degree of present-day RG activity in a particular cluster. It is also to be noted that for small groups, which typically contain only a few large elliptical members, the LO96 fractional local LF nominally implies RG numbers less than one. This is to be interpreted as the present-day probability of observing RG activity in these systems; e.g. for a mass range in which 0.1 RGs are implied, one should see only 1 out of 10 groups to harbor a RG. However, the occurrence of FRII RGs increases dramatically at high $z$, and we expect that all sufficiently large ellipticals have hosted an FRII RG at some point in their lives (e.g. LO96).

We also mention that X-ray observations of a number of presently active RGs inside nearby clusters, including recent high-resolution data by Chandra, do not show any evidence of the RG heating the external medium (Fabian 2001 and references therein). However, one must remember that nearly all such nearby objects, including M87 in Virgo or 3C84 in Perseus, are relatively lowpower, FRI-type RGs, which are presumably subsonic on large scales and incapable of shock heating the ambient ICM (e.g. Bicknell 1995); such RGs were not included in our evaluation above. FRII RGs in dense environments are known to be quite rare in the local universe, with the one exception, Cyg A, displaying good evidence for a bow shock being driven into the ICM, compressing and heating it (Carilli, Perley \& Dreher 1988, Carilli, Perley \& Harris 1994, Clarke, Harris \& Carilli 1997, Wilson, Young \& Shopbell 2000). The prominence of FRII RGs at high redshifts does not entail any contradictions in the role of RGs in shock heating the ICM with observations of nearby cluster RGs.

\subsection{On Assumptions and Uncertainties}

We have obtained numbers for the excess energy input of $\epsilon_{\mathrm{ICM}} \sim 1 \mathrm{keV} /$ particle using what we deem to be the most plausible assumptions and reasonable parameter values. Some remarks concerning these are in order.

We first call attention to three key assumptions used in the above evaluation: 1) the $L_{j}-L_{151}$ cor- 
relation is independent of $z ; 2$ ) the redshift evolution of RGs in clusters is the same as that for the whole RG population; and 3) the fractional RG LF is the same for all clusters. Assumption 1) is consistent with inferences drawn by Blundell et al. (1999), who find no systematic epoch-dependence in the gaseous environments of the $\mathrm{RG}$ population as a whole. Recent investigations of the clustering environments of RGs (e.g. Wold et al. 2000, McLure \& Dunlop 2001) do not point to a strong influence of the environment on the evolutionary properties of RGs, compatible with assumptions 1) and 2). For assumption 3), the Ledlow-Owen fractional LF is independent of cluster richness or morphological type within in their sample of Abell clusters (LO95), and it is not unreasonable to expect that this extends down to the scale of small groups. These are certainly no proofs, but lend some credence to our assumptions.

Of the parameters having direct impact on $\epsilon_{\mathrm{ICM}}$, we have used observationally determined numbers for $f_{j}$ and $N_{E} / M_{g}$. Instead, $\xi_{s}$ was evaluated theoretically, and there is one uncertain aspect which can increase this value. Our discussion above was limited to heating of the ICM by the forward shocks induced by RGs, but as mentioned in Sec.2.2, the better part of the total RG energy release should be stored inside its cocoon as internal energy of jet plasma. Although it is believed that the the cocoons of FRII RGs are overpressured and their interior segregated from the ICM during much of the RGs' lifetime (Begelman \& Cioffi 1989), it is unclear what occurs after the activity ceases. One possibility is that cocoons mostly dissipate after reaching pressure equilibrium with the ambient ICM due to instabilities operating at the cocoon-ICM interface (or perhaps even earlier, Reynolds, Heinz \& Begelman 2000). Alternatively, it has been proposed that cocoons may retain their entity for a long time afterwards, perhaps until the host cluster undergoes a major merger (EK00). If the cocoon dissipates, its internal energy, amounting to $75 \%(60 \%)$ of the total RG energy release for relativistic (non-relativistic) cocoon plasma, can be released into the ICM, and any fraction that is thermalized with the ICM will add to the excess energy and raise $\xi_{s}$. The actual amount that can contribute to $\epsilon_{\mathrm{ICM}}$ in this way is difficult to assess, as it depends on the highly uncertain composition, particle distribution, and magnetic field content of the jet plasma, but in any case, we should bear in mind the fact that the $\mathrm{RG}$ cocoon is a significant energy reservoir.

\subsection{Comparison with Supernovae Heat- ing}

As discussed in Sec.1, the substantial amounts of heavy elements observed in the ICM certifies that SNe must have affected the ICM in one way or other, but this does not necessarily imply that they have been important energetically. One may envision ways of polluting the ICM with metals without recourse to SN-driven galactic winds, such as ram-pressure stripping, repeated tidal interactions ('galaxy harassment'), mergers, etc. Theoretically, it is difficult to conceive how the combined kinetic energy of an ensemble of SNe can be ejected from their host galaxies and then deposited to the ICM with high efficiency; heavy radiative losses during this process seem to be unavoidable (Kravtsov \& Yepes 2000, Wu et al. 2000).

However, some observations of SN-driven winds in starburst galaxies suggest a rather high efficiency of energy conversion (Heckman 2000), and there is room for speculation that $\mathrm{SNe}$ do partake in the energetics of the ICM. Sasaki (2001) has also suggested the possibility of intracluster SNe, i.e. those which explode outside galaxies and directly heat the ICM. It is then preferable to have some independent observational test which can discriminate between our proposal of RGs heating the ICM and that by SNe.

In principle, one possibility involves the redshift evolution of the X-ray scaling relations. The space density of FRII RGs is seen to increase with redshift faster than the average star formation rate (approximately equal to the SN rate) in the universe (e.g. Madau 1999 and references therein), and this may be reflected in the $z$ evolution of the $\mathrm{L}-\mathrm{T}$ and $\mathrm{S}-\mathrm{T}$ relations. At present the $\mathrm{L}-\mathrm{T}$ relation is available only out to $z \sim 0.4$ and only with large errors (Mushotzky \& Scharf 1997), but the observational situation should improve significantly in the near future with Chandra and XMM-Newton observations. However, the expected difference between RGs and SNe may not be too drastic, and could render this prospect impractical.

One would appreciate some observational signature which can directly identify RGs in the process 
of heating the ICM at the expected epochs. The long radiative cooling time of the shocked ICM means that the thermal X-ray emission from this gas never becomes very luminous and could be difficult to observe. However, the same shocks which heat the ICM should also generate non-thermal particles through shock acceleration, and the associated high energy radiation, particularly from relativistic electrons, can offer a potent diagnostic, which we discuss next.

\subsection{Non-thermal Gamma-ray Emission}

Non-thermal gamma-rays emitted by shock accelerated electrons hold the promise of being a very direct and powerful probe of large-scale shock heating processes in the ICM/IGM. Strong shocks, which are a requisite element in our picture of ICM heating, are also known to be very conducive to non-thermal particle acceleration via the first order Fermi mechanism (Blandford \& Eichler 1987 and references therein). Relativistic electrons accelerated and injected into the shocked ICM subsequently lose energy by emitting synchrotron and inverse Compton (IC) radiation, the latter mainly by upscattering cosmic microwave background photons. For typical ICM magnetic fields $\left(B_{\mathrm{ICM}} \sim 0.1-1 \mu \mathrm{G}\right)$, IC dominates, and much of the electrons' energy should end up as gammarays. The crucial point of note is that at sufficiently high energies, relativistic electrons have IC cooling times much shorter than the duration of the shock (here the RG lifetime), so that the resultant gamma-ray emission is 'prompt' and directly traces the period of strong shock activity. Such gamma-rays therefore serve as beacons, enabling us to 'see' the moment at which large-scale shocks are occurring. The significance of such prompt gamma-ray emission was recognized by Loeb \& Waxman (2000) and Totani \& Kitayama (2000), in the context of gravitational shocks in groups and clusters. Gamma-rays of gravitational shock origin may be suppressed if, as in our RG scenario, the pre-collapse IGM of groups and clusters has been substantially heated by non-gravitational sources (Totani \& Inoue 2001).

For shocks induced by the RGs themselves, the typical gamma-ray spectra and luminosities can be estimated as follows. A fraction $\xi_{e}$ of the RG kinetic power $L_{j}$ is assumed to be converted to non-thermal electrons with a power- law distribution $d n / d \gamma \propto \gamma^{-p}, \gamma$ being the electron Lorentz factor. The maximum Lorentz factor $\gamma_{\max }$ is set by equating the shock acceleration time with the IC cooling time, $\gamma_{\max } \sim 1.2 \times$ $10^{9}(1+z)^{-2}\left(B_{\mathrm{ICM}} / 1 \mu \mathrm{G}\right)^{1 / 2}\left(V_{s} / 10^{4} \mathrm{kms}^{-1}\right)$, where $V_{s} \sim 10^{4} \mathrm{kms}^{-1}$ is a typical velocity of the forward shock ( $\simeq$ the hot spot advance speed) for an FRII RG. The cooling Lorentz factor $\gamma_{c}$ is where the IC cooling time equals the RG lifetime $\tau_{\mathrm{RG}} \sim$ $10^{8} \mathrm{yr}, \gamma_{c} \sim 2.3 \times 10^{4}(1+z)^{-4}\left(\tau_{\mathrm{RG}} / 10^{8} \mathrm{yr}\right)^{-1}$, and above this Lorentz factor all electrons cool within the shock duration. The emission energies corresponding to $\gamma_{c}$ and $\gamma_{\max }$ are respectively $E_{c} \sim$ $450 \mathrm{keV}(1+z)^{-7}\left(\tau_{\mathrm{RG}} / 10^{8} \mathrm{yr}\right)^{-2}$ and $E_{\max } \sim 1.2 \times$ $10^{3} \mathrm{TeV}(1+z)^{-3}\left(B_{\mathrm{ICM}} / 1 \mu \mathrm{G}\right)\left(V_{s} / 10^{4} \mathrm{kms}^{-1}\right)^{2}$, between which the spectrum will have a cooled energy index $\alpha_{c}=p / 2$. Below $\gamma_{c}$ the electrons are mostly adiabatic, and the energy index of the spectrum below $E_{c}$ is $\alpha_{a d}=(p-1) / 2$.

Strong shocks should result in $p \sim 2$ and hence $\alpha_{a d} \sim 0.5$ and $\alpha_{c} \sim 1$, i.e. constant luminosity per logarithmic energy interval above $E_{c}$. The efficiency of electron injection $\xi_{e}$ in astrophysical shocks is a critical but rather uncertain parameter, both observationally and theoretically. For shocks in supernova remnants, this is believed to be somewhere in the range $\xi_{e} \sim 0.001-0.05$ (e.g. Sturner et al. 1997, Baring et al. 1999, Tanimori et al. 1998). It may also be more appropriate to measure $\xi_{e}$ with respect to the thermal energy of the post-shock gas rather the total kinetic energy, in which case we must also account for $\xi_{s}$, the efficiency of energy input into the ICM. Although our fiducial choice will be $\xi_{e}=0.05$, we caution that this number could be lower in reality, and our estimates below more on the optimistic side. We do note that recent observations of hard X-ray tails in groups and clusters (e.g. Rephaeli 2001), if interpreted as non-thermal emission from electrons, seem to require high electron injection efficiencies, of order $\xi_{e}=0.05$ (e.g. Sarazin 1999, Takizawa \& Naito 2000, Blasi 2001).

A RG at $z$ will be observed to have an integral gamma-ray flux above energy $E_{*}$

$$
F_{*} \sim \frac{1+z}{E_{*} \ln \left(\gamma_{\max }\right)} \frac{\xi_{e} L_{j}}{4 \pi d_{L}(z)^{2}},
$$

where $d_{L}(z)$ is the luminosity distance (c.f. Totani $\&$ Kitayama 2000). The nearest, luminous FRII $\mathrm{RG}$ Cyg A lies in a relatively rich cluster at 
$z=0.058$, and should possess a jet power $L_{j} \sim$ $3 \times 10^{46} \mathrm{ergs}^{-1}$. In the lambda cosmology, the high-energy gamma-ray flux above $100 \mathrm{MeV}$ from this object is estimated to be $F_{100} \sim 8 \times 10^{-8}$ photons $\mathrm{cm}^{-2} \mathrm{~s}^{-1}$, below the $2 \sigma$ upper limit ascertained by the EGRET instrument, $1.7 \times 10^{-7}$ photons $\mathrm{cm}^{-2} \mathrm{~s}^{-1}$ (Fichtel et al. 1994). However, this is easily detectable by the GLAST satellite, whose nominal sensitivity for a 2 year all-sky survey is $2 \times 10^{-9}$ photons $\mathrm{cm}^{-2} \mathrm{~s}^{-1}$ above 100 $\mathrm{MeV}$ (Gehrels \& Michelson 1999); other relatively nearby FRII RGs should be observable as well. The more luminous high-redshift RGs with radio luminosities $L_{151} \gtrsim 10^{28} \mathrm{~W} \mathrm{~Hz}^{-1} \mathrm{sr}^{-1}$ and corresponding jet powers $L_{j} \gtrsim 2.7 \times 10^{47} \mathrm{ergs}^{-1}$ would be within the capabilities of GLAST even at $z=1$, allowing the era of substantial ICM heating to be directly probed.

Using the W01 radio LFs, it is also straightforward to evaluate the gamma-ray source counts for the whole population. The number of RGs with gamma-ray flux greater than $F_{*}$ is

$$
\begin{aligned}
N\left(>F_{*}\right)=\int d z \frac{d V}{d z} \int_{\max \left\{L_{151}\left(F_{*}, z\right), L_{151, \min }\right\}}^{L_{151, \max }} & \times d \log L_{151} \rho\left(L_{151}, z\right),
\end{aligned}
$$

where $L_{151}\left(F_{*}, z\right)$ is the $151 \mathrm{MHz}$ radio luminosity of a RG at $z$ that would produce a flux $F_{*}$, and $\frac{d V}{d z}$ is the comoving volume element of the universe. Fig. 4 shows the predicted $\log N-\log F_{100}$ distribution of gamma-ray emitting RGs. For the lambda cosmology, we predict that GLAST would see about 20 discrete gamma-ray sources which are non-variable and associated with powerful RGs during a 2 year all-sky survey. Since these RGs are strong radio emitters as well, targeted observations of selected objects could do even better; for example, at a sensitivity of $10^{-9}$ photons $\mathrm{cm}^{-2} \mathrm{~s}^{-1}$ (just a factor of 2 better than the 2 year survey limit), up to 70 RGs are detectable.

On the other hand, the number of observable RGs should have been negligible for EGRET. This indicates that RGs are unlikely to have contributed greatly to any of the unidentified EGRET sources (Hartman et al. 1999) or the extragalactic gamma-ray background (Sreekumar et al. 1998).

It is emphasized that besides the forward shock in the ICM, electron acceleration should also be conspicuous at the reverse shock inside the radio lobes, i.e. the hot spots. However, copious high energy gamma-ray emission is expected only from the forward shock; since the magnetic fields in the hot spots are much higher than in the ICM (e.g. Meisenheimer et al. 1989), synchrotron cooling dominates and forces the maximum emission energy of the reverse shock electrons to be well below the GLAST energy band. This makes the above test all the more effective: high energy gammaray emission does not merely signal the presence of a powerful RG per se, but necessitates that the external medium is being actively shocked.

The gamma-ray diagnosis should be markedly different if ICM heating were due to SN-driven winds. The kinetic powers of individual winds is probably at most $L_{w} \sim 10^{42}-10^{43} \mathrm{erg} \mathrm{s}^{-1}$, and their lifetimes may be around $\tau_{w} \sim 10^{7} \mathrm{yr}$ (Heckman 2000). Thus the consequent non-thermal emission by shock-accelerated electrons of SNdriven wind origin is much too weak to be detectable as discrete gamma-ray sources, barring the highly unlikely situation of $10^{3}$ to $10^{4}$ such winds being active nearly simultaneously (i.e. within $\tau_{w}$ ). These statements apply to intracluster $\mathrm{SNe}$ as well, whose explosion times should be even less correlated with each other. Thus future gamma-ray observations offer good prospects for directly corroborating that RGs indeed shock heat the ICM at the expected epochs, as well as discriminating between the $\mathrm{SNe}$ scenario.

We further remark that the same shocks which accelerate electrons should do so for protons as well, perhaps with an even higher injection efficiency. These non-thermal protons can undergo inelastic collisions with thermal protons of the ICM gas, and thereby emit high energy gammarays via neutral pion decay (e.g. Dar \& Shaviv 1995, Völk, Aharonian \& Breitschwerdt 1996, Berezinsky, Blasi \& Ptuskin 1997), or through IC radiation by secondary electrons (e.g. Blasi \& Colafrancesco 1999). As the cooling times of such high energy protons are generally longer than the Hubble time (Enßlin et al. 1997), protoninduced gamma-ray emission is neither efficient nor prompt, and would not serve as useful 'signposts' of the shock heating epoch. On the other hand, their confinement times in the ICM are also expected to be very long (Völk et al. 1996, Berezinsky et al. 1997), so any non-thermal protons injected into the ICM by various kinds of 
shock activity may accumulate over the lifetime of a cluster, and the accompanying emission should carry important information on its entropy history. We wish to explore this interesting issue of non-thermal protons in relation to nongravitational mechanical feedback processes in the IGM/ICM in a future paper.

\subsection{Non-thermal X-ray Emission and Other Implications}

We briefly remark on some additional implications.

Following the above discussion, we note that relativistic electrons accelerated at the ICM shock should also emit non-thermal X-ray emission. These X-rays will not be as effective as gammarays in offering a test of our proposal, since 1) the emitting electrons have cooling times longer than the shock duration, so most of the emission is not 'prompt' and delayed with respect to the RG activity, and 2) confusion with other Xray components, such as emission from the $\mathrm{RG}$ hotspots and knots, the nucleus, as well as from the undisturbed ICM, is problematic. Conversely, non-thermal X-ray emission is interesting in that it may last for a relatively long time after the shock activity and particle injection has ceased, and the implications of such 'relic' X-ray emission has been discussed in several recent papers (e.g. Sarazin 1999, Atoyan \& Völk 2000, Blasi 2001) in response to possible detections of hard X-ray tails in rich clusters (Rephaeli 2001 and references therein). With regard to our study, we call attention to recent reports of excess hard $\mathrm{X}$-ray emission in the nearby group of galaxies HCG62 by Fukazawa et al. (2000), and similar detections for other groups by Nakazawa (2001, private communication). In any non-gravitational heating scenario which envisions significant shock heating of group gas prior to its formation, the gravitational shock accompanying the subsequent collapse is strongly suppressed (e.g. Balogh et al. 1999, Tozzi \& Norman 2001). Non-thermal particle acceleration should result only from the heating sources themselves, which are RGs in our case. The radio emission from the RG itself could fade rather rapidly after its death (Goldshmidt \& Rephaeli 1994, EK00), and may not leave a visible trace of past RG activity. If interpreted as nonthermal emission, the X-ray excesses could then be the 'smoking gun' of shock heating by RGs in the not-too-remote past for these particular groups.

A different type of non-thermal emission may also arise, which would be peculiar to RGs and related to the issue of cocoon dissipation (Sec.3.2) As already mentioned, the exact constituents of the jet plasma inside the cocoon are unknown, but it is quite possible that it comprises primarily relativistic, non-thermal particles, whose energy content can be very large. Cocoon particles of this kind injected into the ICM may give forth to observable non-thermal features distinct from the forward shock emission, at much later epochs. Although such processes cannot be reliably predicted at the moment, this possibility needs to be explored in the future.

Finally, as suggested by Valageas \& Silk (1999) for active galaxies in general, we speculate that the large energy input into the ICM by RGs may have played some role in regulating the formation and evolution of stars and galaxies inside clusters.

\section{Conclusions}

We conclude by summarizing the salient points addressed in this paper.

Utilizing recently published radio LFs (W01) and the observed correlation between jet power and radio luminosity (Rawlings 1992, W99), the total energy output of RG jets in the universe can be evaluated with reasonable confidence, $U_{j} \sim$ $1-3 \times 10^{57} \mathrm{ergMpc}^{-3}$, the bulk of which is attained at redshifts $z \sim 1-3$. Combining this with the local RG LF in clusters (LO95, LO96), the total energy input into the ICM can be estimated to be $1-2 \mathrm{keV}$ per particle, precisely in the range recent models require to reproduce the observed deviations from self-similarity in the luminositytemperature relation of groups and clusters. RGs have a decided edge over $\mathrm{SNe}$ in that they directly heat the ICM outside galaxies and are immune to radiative cooling. The picture of ICM shock heating by RGs may be most clearly tested and distinguished from that due to $\mathrm{SNe}$ by observing the high energy gamma-rays emitted by shock-accelerated electrons during the era of ICM heating, which can be realized by GLAST.

S.I. is grateful to a number of colleagues for valuable discussions, particularly T. Kitayama, as 
well as M. Nagashima, Y. Fujita and T. Totani. This research was supported by the Grant-in-Aid for Scientific Research from the Ministry of Education, Science, Sports and Culture of Japan (No. 12304009).

\section{REFERENCES}

Arnaud, M. \& Evrard, A. E. 1999, MNRAS, 305, 631

Atoyan, A. M. \& Volk, H. J. 2000, ApJ, 535, 45

Balogh, M. L., Babul, A. \& Patton, D. R. 1999, MNRAS, 307, 463

Baring, M. G., Ellison, D. C., Reynolds, S. P., Grenier, I. A. \& Goret, P. 1999, ApJ, 513, 311

Begelman, M. C. \& Cioffi, D. F. 1989, ApJ, 345, L21

Berezinsky, V. S., Blasi, P. \& Ptuskin, V. S. 1997, ApJ, 487, 529

Bicknell, G. V. 1995, ApJS, 101, 29

Blandford, R. D. \& Eichler, D. 1987, Phys. Rep., 154,1

Blasi, P. 2001, Astropart. Phys., 15, 223

Blasi, P. \& Colafrancesco, S. 1999, Astropart. Phys., 12, 169

Blundell, K. M. \& Rawlings, S. 2000, AJ, 119, 1111

Blundell, K. M., Rawlings, S. \& Willott, C. J. 1999, AJ, 117, 677

Bower, R. G., Benson, A. J., Baugh, C. M., Cole, S., Frenk, C. S. \& Lacey, C. G. 2000, astro$\mathrm{ph} / 0006109$

Bryan, G. L. 2000, ApJ, 544, L1

Bryan, G. L. \& Voit, G. M. 2001, astroph/0101467

Carilli, C. L. \& Barthel, P. D. 1996, A\&A Rev., 7, 1

Carilli, C. L., Perley, R. A. \& Dreher, J. W. 1988, ApJ, 334, L73
Carilli, C. L., Perley, R. A. \& Harris, D. E. 1994, MNRAS, 270, 173

Cavaliere, A., Giacconi, R. \& Menci, N. 2000, ApJ, 528, L77

Cen, R. \& Ostriker, J. P. 1999, ApJ, 514, 1

Clarke, D. A., Harris, D. E. \& Carilli, C. L. 1997, MNRAS, 284, 981

Dar, A. \& Shaviv, N. J. 1995, Phys. Rev. Lett., 75,3052

David, L. P., Slyz, A., Jones, C., Forman, W., Vrtilek, S. D. \& Arnaud, K. 1993, ApJ, 479, 488

Davé, R., Cen, R., Ostriker, J. P., Bryan, G. L., Hernquist, L., Katz, N., Weinberg, D. H., Norman, M. L. \& O'Shea, B. 2000, astro$\mathrm{ph} / 0007217$

Dunlop, J. S. \& Peacock, J. A. 1990, MNRAS, 247,19

Eke, V. R., Navarro, J. F. \& Frenk, C. S. 1998, ApJ, 503, 569

Enßlin, T. A., Biermann, P. L., Kronberg, P. P. \& Wu, X.-P. 1997, ApJ, 477, 560

Enßlin, T. A. \& Kaiser, C. R. 2000, A\&A, 360, 417 (EK00)

Enßlin, T. A., Wang, Y., Nath, B. B. and Biermann, P. L. 1998, A\&A, 333, L47

Evrard, A. E. \& Henry, J. P. 1991, ApJ, 383, 95

Fabian, A. C. 2001, astro-ph/0103392

Fanaroff, B. L. \& Riley, J. M. 1974, MNRAS, 167, $31 \mathrm{P}$

Fichtel, C. E. et al. 1994, ApJS, 94, 551

Finoguenov, A., David, L. P. \& Ponman, T. J. 2000, ApJ, 544, 188

Fukazawa, Y. et al. 2001, ApJ, 546, L87

Fukugita, M., Hogan, C. J. \& Peebles, P. J. E. 1998, ApJ, 503, 518

Fusco-Femiano, R. \& Hughes, J. P. 1994, ApJ, 429,545 
Gehrels, N. \& Michelson, P. 1999, Astropart. Phys., 11, 277

Goldshmidt, O. \& Rephaeli, Y. 1994, ApJ, 431, 586

Hardcastle, M. J. \& Worrall, D. M. 2000, MNRAS, 319,562

Hartman, R. C. et al. 1999, ApJS, 123, 79

Heckman, T. M. 2000, astro-ph/0009075

Heinz, S., Reynolds, C. S. \& Begelman, M. C. 1998, MNRAS, 501, 126

Helsdon, S. F. \& Ponman, T. J. 2000, MNRAS, 315,356

Kaiser, C. R. \& Alexander, P. 1999, MNRAS, 305, 707

Kaiser, C. R., Dennett-Thorpe, J. \& Alexander, P. 1997, MNRAS, 292, 723

Kaiser, N. 1986, MNRAS, 222, 323

Kaiser, N. 1991, ApJ, 383, 104

Kitayama, T. \& Suto, Y. 1996, ApJ, 469, 480

Kravtsov, A. V. \& Yepes, G. 2000, MNRAS, 318, 227

Lacey, C. G. \& Cole, S. 1993, MNRAS, 262, 627

Leahy, J. P. \& Gizani, N. A. B. 1999, astro$\mathrm{ph} / 9909121$

Ledlow, M. J. \& Owen, F. N. 1995, AJ, 109, 853 (LO95)

Ledlow, M. J. \& Owen, F. N. 1996, AJ, 112, 9 (LO96)

Lloyd-Davies, E. J., Ponman, T. J. \& Cannon, D. B. 2000, MNRAS, 315, 689

Loeb, A. \& Waxman, E. 2000, Nature, 405, 156

Madau, P. 1999, astro-ph/9902228

Markevitch, M. 1998, ApJ, 504, 27

McLure, R. J. \& Dunlop, J. S. 2001, MNRAS, 321, 515
Meisenheimer, K., Röser, H.-J., Hiltner, P. R., Yates, M. G., Longair, M. S., Chini, R. \& Perley, R. A. 1989, A\&A, 219, 63

Menci, N. \& Cavaliere, A. 2000, MNRAS, 311, 50

Mushotzky, R. F. \& Scharf, C. A. 1997, ApJ, 482, L13

Pen, U.-L. 1999, ApJ, 510, L1

Ponman, T. J., Bourner, P. D. J., Ebeling, H. \& Böhringer, H. 1996 MNRAS, 283, 690

Ponman, T. J., Cannon, D. B. and Navarro, J. F. 1999, Nature, 397, 135

Rawlings, S. 1992, in Extragalactic Radio Sources: From Beams to Jets, eds. Roland, J., Sol, H. \& Pelletier, G., Cambridge University Press

Rawlings, S. 2000, astro-ph/0008067

Rawlings, S. \& Saunders, R. 1991, Nature, 349, 138

Renzini, A. 2000, astro-ph/0001312

Rephaeli, Y. 2001, astro-ph/0101363

Reynolds, C. S., Heinz, S. \& Begelman M. C. 2001, ApJ, 549, L179

Sarazin, C. L. 1999, ApJ, 520, 529

Sasaki, S. 2001, PASJ, 53, 53

Sreekumar, P. et al. 1998, ApJ, 494, 523

Sturner, S. J., Skibo, J. G., Dermer, C. D. \& Mattox, J. R. 1997, ApJ, 490, 619

Takizawa, M. \& Naito, T. 2000, ApJ, 535, 586

Tanimori, T. et al. 1998, ApJ, 497, L25

Thompson, L. A. \& Gregory, S. A. 1980, ApJ, 242, 1

Totani, T. \& Inoue, S. 2001, astro-ph/0104072, to appear in Astropart.Phys.

Totani, T. \& Kitayama. T. 2000, ApJ, 545, 572

Tozzi, P. \& Norman, C. 2001, ApJ, 546, 63

Tozzi, P., Scharf, C. \& Norman, C. 2000, ApJ, 542, 106 
Urry, C. M. \& Padovani, P. 1995, PASP, 107, 803

Valageas, P. \& Silk, J. 1999, A\&A, 350, 725

Venturi, T., Giovannini, G. \& Feretti, L. 1990, AJ, 99,1381

Völk, H. J., Aharonian, F. A. \& Breitschwerdt, D. 1996, Space Sci. Rev., 75, 279

Voit, G. M. \& Bryan, G. L. 2001, ApJ, 551, L139

Willott, C. J., Rawlings, S., Blundell, K. M. \& Lacy, M. 1999, MNRAS, 309, 1017 (W99)

Willott, C. J., Rawlings, S., Blundell, K. M., Lacy, M. \& Eales, S. A. 2001, MNRAS, 322, 536 (W01)

Wilson, A. S., Young, A. J. \& Shopbell, P. L. 2001, astro-ph/0101422

Wold, M., Lacy, M., Lilje, P. B. \& Serjeant, S. 2000, MNRAS, 316, 267

Wu, K. K. S., Fabian, A. C. \& Nulsen, P. E. J. 1998, MNRAS, 301, L20

Wu, K. K. S., Fabian, A. C. \& Nulsen, P. E. J. 1999, astro-ph/9910122

Wu, K. K. S., Fabian, A. C. \& Nulsen, P. E. J. 2000, MNRAS, 318, 889

Yamada, M. \& Fujita, Y. 2001, astro-ph/0105102

This 2-column preprint was prepared with the AAS LATEX macros v5.0.

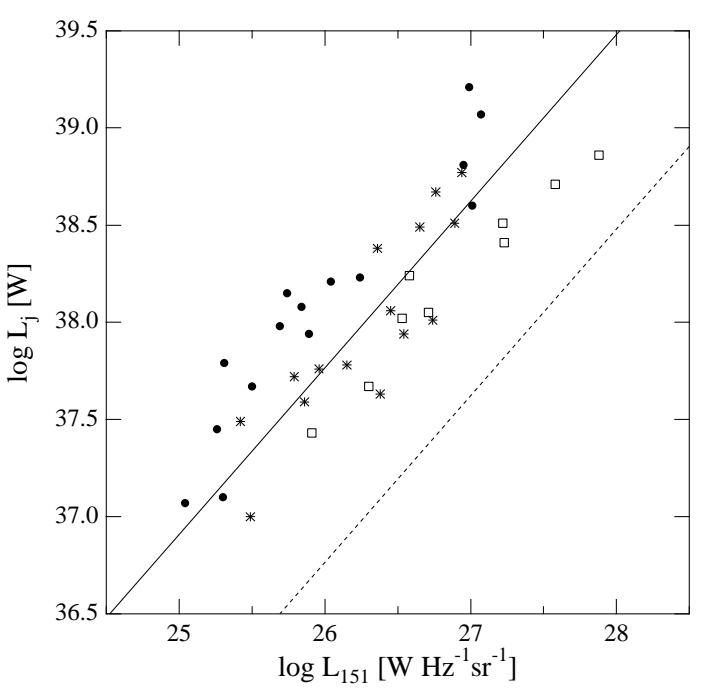

Fig. 1. - The observed correlation between $L_{151}$ and $L_{j}$ assuming $f_{j}=1$ and $h_{50}=1$, from Rawlings (1992). RGs in isolated $\left(n \lesssim 10^{-5} \mathrm{~cm}^{-3}\right)$, group $\left(10^{-5} \mathrm{~cm}^{-3} \lesssim n \lesssim 10^{-3} \mathrm{~cm}^{-3}\right)$, and cluster $\left(n \gtrsim 10^{-3} \mathrm{~cm}^{-3}\right)$ environments are indicated by circles, asterisks and squares, respectively. Our adopted correlation of eq. 1 is indicated by the solid line, and that in Willott et al. (1999) by the dashed line. 

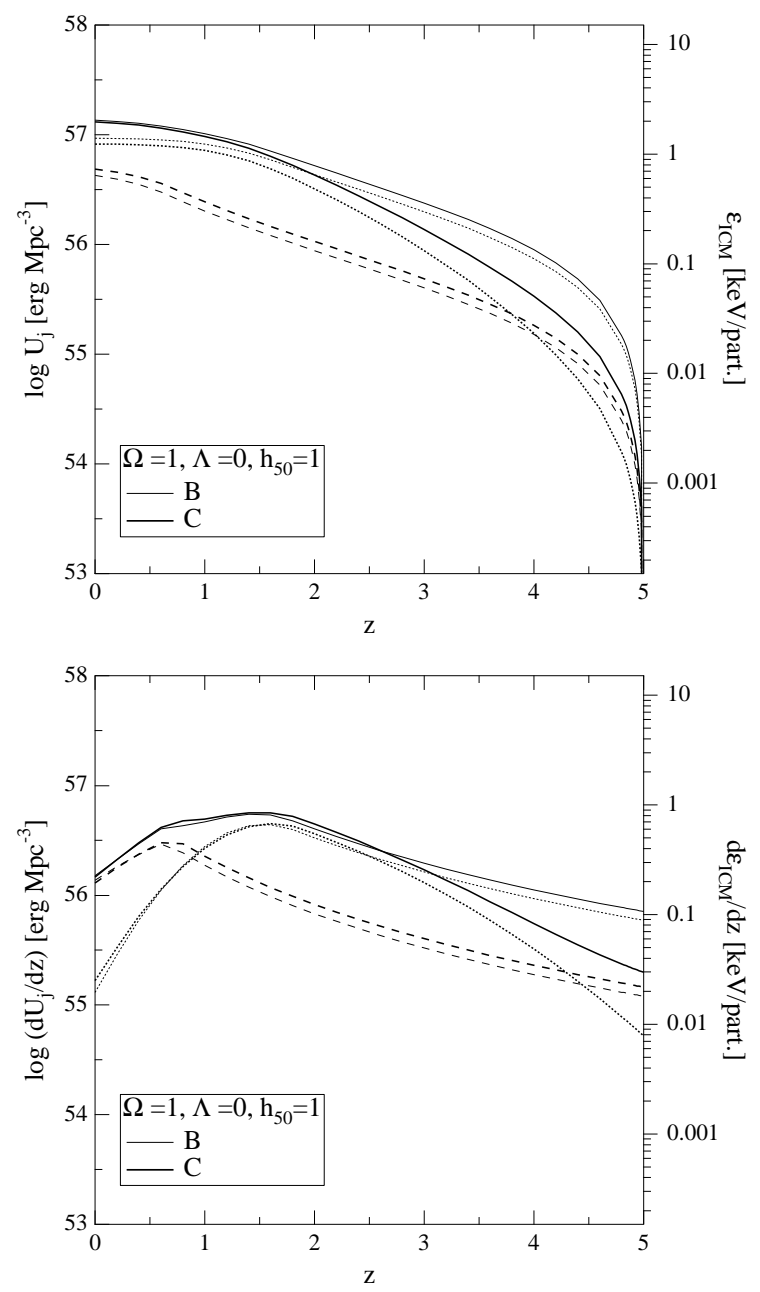

Fig. 2.- a) The cumulative jet energy output from $z_{\max }=5$ up to $z$, and b) The differential jet energy output per unit $z$, for model LFs B (thin) \& C (thick) of Willott et al. (2001) in the case of $\Omega=1, \Lambda=0$ and $h_{50}=1$. The contributions of the low-luminosity population (dashed) and the high-luminosity population (dotted) are shown separately along with their sum (solid).
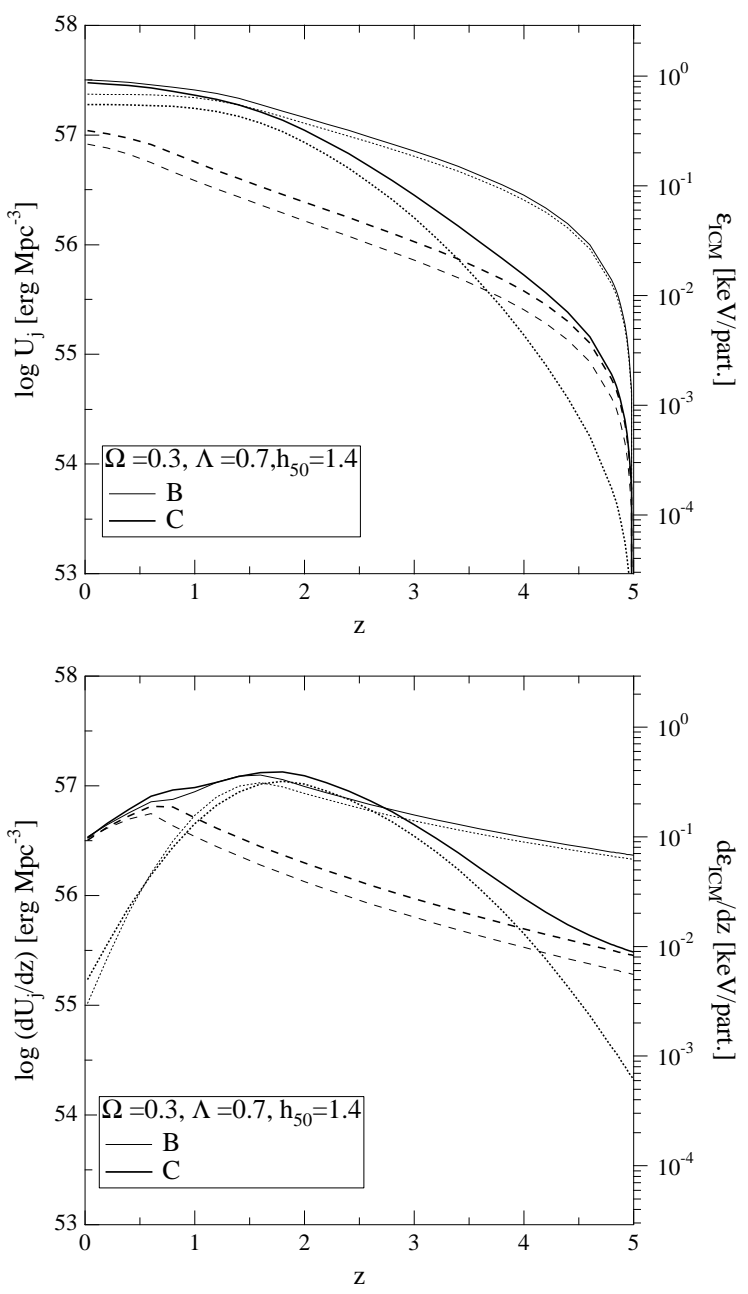

Fig. 3.- Same as Fig.2, but for $\Omega=0.3, \Lambda=0.7$ and $h_{50}=1.4$. 


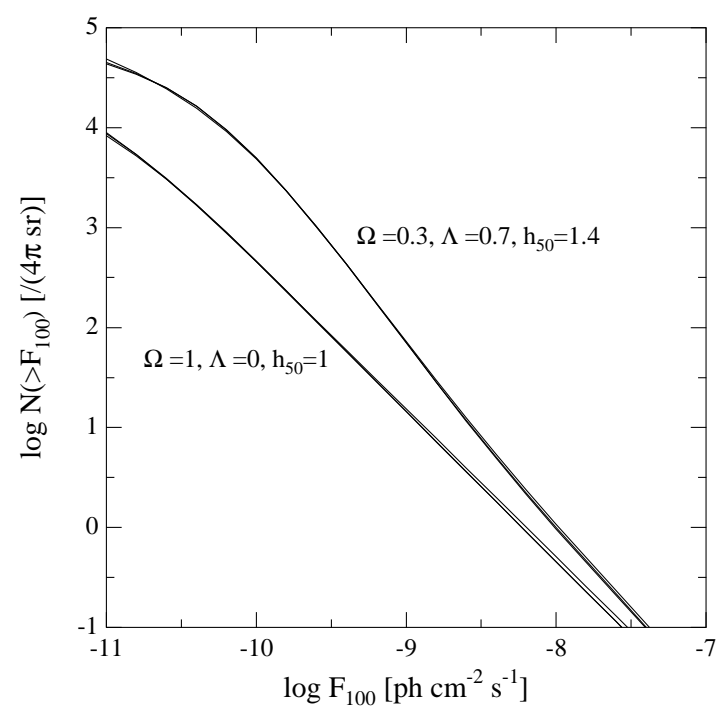

Fig. 4.- The cumulative all-sky number of radio galaxies with gamma-ray flux larger than $F_{100}$. Curves are shown for all model LFs (A, B and C), but are almost degenerate within either cosmology, EdS (bottom) and lambda (top). 